\title{
GENERIC AFFINE DIFFERENTIAL GEOMETRY OF PLANE CURVES
}

\author{
by SHYUICHI IZUMIYA and TAKASI SANO
}

(Received 23rd July 1996)

\begin{abstract}
We study affine invariants of plane curves from the view point of the singularity theory of smooth functions
\end{abstract}

1991 Mathematics subject classification: 53A15, 58C27.

\section{Introduction}

There are several articles which study "generic differential geometry" in Euclidean space $([2,3,4,5,6,7$, etc]). The main tools in these articles are the distance-squared function and the height function. The classical invariants of extrinsic differential geometry can be treated as "singularities" of these functions, however, as Fidal [7] pointed out, the geometric interpretation of sextactic points of a convex curve is quite complicated from this point of view. We say that a point $p$ of a convex curve $C$ is a sextactic point if there exists a conic touching $C$ at $p$ with at least six-point contact.

On the other hand, it has been classically known that a sextactic point of a convex curve corresponds to a stationary point of affine curvature (i.e., so called "affine vertex") in affine differential geometry (cf., $[1,8,9])$. We also say that a point $p$ is $a$ parabolic point if there exists a unique parabola touching $C$ at $p$ with five-point contact which is known as a zero point of the affine curvature (i.e., so called "affine inflexion"). In this paper we introduce the new notions of affine distance-cubed functions and affine height functions of a convex curve. These functions are quite useful for the study of generic properties of invariants of the extrinsic affine differential geometry of convex plane curves.

As a consequence, we can apply ordinary techniques of the singularity theory for these functions and get information on sextactic points and parabolic points of a convex plane curve. This not only simplifies the arguments about sextactic points but also gives a new interpretation of parabolic points. In Section 2 we shall introduce the notion of the affine normal curve of a convex plane curye. We shall show that a cusp singular point of the affine normal curve corresponds to a parabolic point of the original curve (cf., Figure 1a; $\gamma(t)=(\cos t(\cos t+2)$, $\sin t(\cos t+2))$ ). 


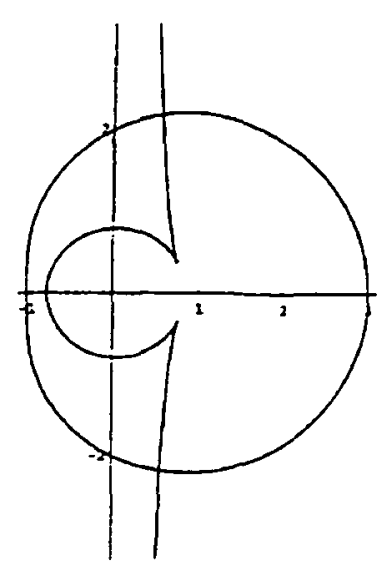

F!GURE 1a

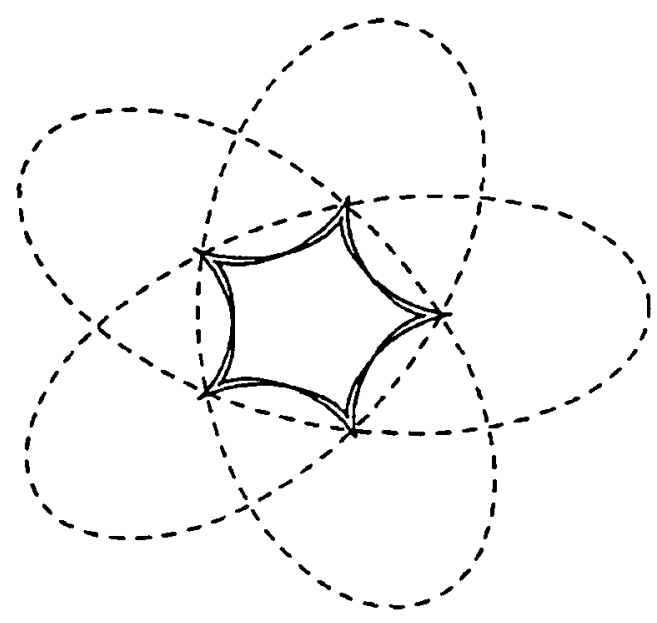

FIGURE lb

Let $\gamma: S^{1} \rightarrow \mathbb{R}^{2}$ be a smooth curve without inflexions. We assume throughout that $\gamma$ has the following properties, both of which are satisfied generically. (We do not prove the fact here (cf., $[6,7])$ ):

(A 1) There is no conic having greater than six-point contact with $\gamma\left(S^{\prime}\right)$.

(A 2) The number of points $p$ of $\gamma\left(S^{1}\right)$ where the unique non-singular conic touching $\gamma\left(S^{1}\right)$ at $p$ with at least five-point contact is a parabola, is finite.

(A 3) There is no parabola having six-point contact with $\gamma\left(S^{1}\right)$.

Under these assumptions, we shall give some interpretations of affine invariants of convex plane curves from the view point of singularity theory. The main result in this paper is Theorem 2.2 which will be given in Section 2. This theorem asserts that an affine vertex (resp. the affine inflexion) corresponds to a cusp point of the affine evolute (resp. the affine normal curve) under the above assumptions (cf., Figure lb; $\gamma(t)=(2 \cos 3 t+\cos 2 t, 2 \sin 3 t-\sin 2 t))$. This assertion for an affine vertex has been already known by Fidal [7]; however, the arguments we use here are incredibly easy compared with Fidal's arguments.

The basic techniques in this paper depend heavily on those in the attractive book of Bruce and Giblin [5], so that the authors are grateful to both of them. The authors also wish to express their hearty thanks to the referee for careful readings.

All curves and maps considered here are of class $C^{\infty}$ unless stated otherwise.

\section{Basic notions}

Let $\mathbb{R}^{2}$ be an affine plane where we adopt coordinates such that the area of the parallelogram spanned by two vectors $a=\left(a_{1}, a_{2}\right), \quad b=\left(b_{1}, b_{2}\right)$ is given by 
$|a, b|=a_{1} b_{2}-a_{2} b_{1}$. We fix the above coordinates in this section. Let $\gamma: I \rightarrow \mathbb{R}^{2}$ be a curve with $|\dot{\gamma}(t), \ddot{\gamma}(t)| \neq 0$ (i.e., without inflexion points), where $\dot{\gamma}(t)=\frac{d \gamma}{d t}(t)$. The affine arc-length of a curve $\gamma$, measured from $\gamma\left(t_{0}\right), t_{0} \in I$ is $s(t)=\int_{t_{0}}^{t}|\dot{\gamma}(t), \dot{\gamma}(t)|^{1 / 3} d t$ (cf., [9, p. 7]). Then a parameter $s$ is determined such that $\left|\gamma^{\prime}(s), \gamma^{\prime \prime}(s)\right|=1$, where $\gamma^{\prime}(s)=\frac{d y}{d s}(s)$. So we say that a curve $\gamma$ is parameterized by affine arc-length if it satisfies $\left|\gamma^{\prime}(s), \gamma^{\prime \prime}(s)\right|=1$.

By arguments similar to those in Euclidean differential geometry, we have the following Frenet-Serret type formula:

$$
\gamma^{\prime \prime \prime}(s)=-k_{a}(s) \gamma^{\prime}(s)
$$

where $k_{a}(s)=\left|\gamma^{\prime \prime}(s), \gamma^{\prime \prime \prime}(s)\right|$ which is called an affine curvature of $\gamma$. We also call $\gamma^{\prime \prime}(s)$ the affine normal vector of $\gamma$. Suppose that $k_{a}(s) \neq 0$, then the point $\gamma(s)+\frac{1}{k_{a}(s)} \gamma^{\prime \prime}(s)$ is called the affine centre of curvature of $\gamma$ at $s$. The affine evolute is defined to be the locus of affine centres of curvature. The curve $\gamma^{n}: I \rightarrow \mathbb{R}^{2}$ contains important geometric information (cf., Theorem 2.2 and Section 5). We call the curve $\gamma^{\prime \prime}: I \rightarrow \mathbb{R}^{2}$ the affine normal curve of $\gamma$. The following fact is classically known (cf., $[1,8,9]$ ).

Proposition 2.1. Under the generic assumption (A 1) and (A 2), we have the following assertions:

(1) $p=\gamma\left(s_{0}\right)$ is a parabolic point of $\gamma(I)$ if and only if $k_{a}\left(s_{0}\right)=0$.

(2) $p=\gamma\left(s_{0}\right)$ is a sextactic point of $\gamma(I)$ if and only if $k_{a}^{\prime}\left(s_{0}\right)=0$.

By Proposition 2.1, there is a parabola having six-point contact with $\gamma(I)$ at $p=\gamma\left(s_{0}\right)$ if and only if $k_{a}\left(s_{0}\right)=k_{a}^{\prime}\left(s_{0}\right)=0$. It is known that the affine evolute is the centre locus of the unique conics touching the curve with five-point contact (cf., $[1,8,9])$.

The main result in this paper is the following theorem.

Theorem 2.2. Let $\gamma: I \rightarrow \mathbb{R}^{2}$ be a curve without inflexions satisfying (A 1)-(A 3). Then:

(1) Let $p$ be a point of the affine evolute of $\gamma$ at $s_{0}$, then, locally at $p$, the affine evolute is

(i) diffeomorphic to a line in $\mathbb{R}^{2}$ if the point $\gamma\left(s_{0}\right)$ is not a sextactic point;

(ii) diffeomorphic to an ordinary cusp in $\mathbb{R}^{2}$ if the point $\gamma\left(s_{0}\right)$ is a sextactic point.

(2) Let $p=\gamma^{\prime \prime}\left(s_{0}\right)$ be a point of the affine normal of $\gamma$, then, locally at $p$, the affine normal curve is

(i) diffeomorphic to a line in $\mathbb{R}^{2}$ if the point $\gamma\left(s_{0}\right)$ is not a parabolic point of $\gamma$;

(ii) diffeomorphic to an ordinary cusp in $\mathbb{R}^{2}$ if the point $\gamma\left(s_{0}\right)$ is a parabolic point of $\gamma$.

The ordinary cusp is a curve which is defined by $C=\left\{\left(x_{1}, x_{2}\right) \in \mathbb{R}^{2} \mid x_{1}^{2}=x_{2}^{3}\right\}$. 


\section{Sextactic points}

In this section we briefly review some properties of sextactic points of a plane curve without inflexions from the view point of the Euclidean differential geometry. We will not use any results in this section; however, we can understand that it is very hard to treat sextactic points from this point of view. We now fix the Euclidean structure on $\mathbb{R}^{2}$ which may be considered as an affine structure.

For any convex regular curve $\gamma$, we have two kinds of parameter with respect to the Euclidean structure. We adopt $t$ as the arclength and $s$ as the affine arclength. Let $k(t)$ be the Euclidean curvature of $\gamma(t)$ which is given by $k(t)=|\dot{\gamma}, \ddot{\gamma}|$, where $\dot{\gamma}=\frac{d y}{d t}$. It follows that $\frac{d t}{d s}=|\dot{\gamma}, \ddot{\gamma}|^{-1 / 3}=k(t)^{-1 / 3}$. In [7] Fidal has given characterization of a sextactic point in terms of the curvature $k(t)$ as follows: $\gamma\left(t_{0}\right)$ is a sextactic point if and only if

$$
36 k\left(t_{0}\right)^{4} \dot{k}\left(t_{0}\right)+40 \dot{k}\left(t_{0}\right)^{3}-45 k\left(t_{0}\right) \dot{k}\left(t_{0}\right) \ddot{k}\left(t_{0}\right)+9 k\left(t_{0}\right)^{2} \dddot{k}\left(t_{0}\right)=0
$$

Therefore it is very hard to study sextactic points from the view point of the Euclidean differential geometry, however, it is known that sextactic points correspond to affine vertices. Since $\frac{d t}{d s}=k(t)^{-1 / 3}$, we have $\frac{d^{2} t}{d s^{2}}=-\frac{1}{3} k(t)^{-5 / 3} \dot{k}(t)$ and $\frac{d^{3} t}{d s^{3}}=\frac{5}{9} k(t)^{-3} \dot{k}^{2}-\frac{1}{3} k(t)^{-2} \ddot{k}(t)$. By the Frenet-Serret formula, we have $|\ddot{\gamma}, \ddot{\gamma}|=k^{3}$. It follows from that we have

$$
k_{a}(s)=k(t)^{4 / 3}-\frac{5}{9} k(t)^{-8 / 3} \dot{k}(t)^{2}+\frac{1}{3} k(t)^{-2 / 3} \ddot{k}(t)
$$

Differentiating both sides of the above equation with respect to $s$, we can show that $k_{a}^{\prime}\left(s_{0}\right)=0$ if and only if $\gamma\left(t_{0}\right)$ is a sextactic point, where $s_{0}=\left(t_{0}\right)$.

We can also represent the affine normal curve in terms of the unit tangent vector $T(t)$ and the unit normal vector $N(t)$ obtained from $T(t)$ by rotating anticlockwise through $\frac{\pi}{2}$. By a simple calculation, we have

$$
\begin{aligned}
\gamma^{\prime \prime}(s) & =k(t)^{-1 / 3} \ddot{\gamma}(t)-\frac{1}{3} k(t)^{-4 / 3} \dot{k}(t) \dot{\gamma}(t) \\
& =k(t)^{-2 / 3} \dot{T}(t)-\frac{1}{3} k(t)^{-5 / 3} \dot{k}(t) T(t)
\end{aligned}
$$

Substituting the Euclidean Frenet-Serret formula $\dot{T}(t)=k(t) N(t)$ into the above equation, we have

$$
\gamma^{\prime \prime}(s)=-\frac{1}{3} k(t)^{-5 / 3} \dot{k}(t) T(t)+k(t)^{1 / 3} N(t)
$$

We cannot directly guess the shape of the affine normal curve from this formula, so that we need some new techniques. 


\section{GENERIC AFFINE DIFFERENTIAL GEOMETRY OF PLANE CURVES 319}

\section{Affine invariant functions}

In this section we introduce two kinds of families of affine invariant functions on a plane curve without inflexions which are useful for the study of the invariants of the curve. Let $\gamma: I \rightarrow \mathbb{R}^{2}$ be a plane curve with $\left|\gamma(s)^{\prime}, \gamma(s)^{\prime \prime}\right|=1$.

4.1. Affine distance-cubed functions. We now define a two parameter family of smooth functions on $I$

$$
F: I \times \mathbb{R}^{2} \rightarrow \mathbb{R}
$$

by

$$
F(s, x)=\left|\gamma^{\prime}(s), \gamma(s)-x\right|
$$

We call $F$ the affine distance cubed function on $\gamma$.

Differentiating $F(s, x)$ with respect to $s$, we have

$$
\begin{aligned}
f_{x}^{\prime}(s) & =\left|\gamma^{\prime \prime}(s), \gamma(s)-x\right| \\
f_{x}^{\prime \prime}(s) & =\left|\gamma^{\prime \prime \prime}(s), \gamma(s)-x\right|-1 \\
f_{x}^{\prime \prime \prime}(s) & =\left|\gamma^{(4)}(s), \gamma(s)-x\right| \\
f_{x}^{\prime \prime \prime}(s) & =\left|\gamma^{(5)}(s), \gamma(s)-x\right|+\left|\gamma^{\prime \prime \prime \prime}(s), \gamma^{\prime}(s)\right|
\end{aligned}
$$

where $f_{x}(s)=F(s, x)$ for any $x \in \mathbb{R}^{2}$.

It follows from these formulae that we have the following proposition.

Proposition 4.1. Let $\gamma: I \rightarrow \mathbb{R}^{2}$ be a convex plane curve with $\left|\gamma^{\prime}(s), \gamma^{\prime \prime}(s)\right|=1$. Then

(a) $f_{x}^{\prime}\left(s_{0}\right)=0$ if and only if there exists $\lambda \in \mathbb{R}$ such that $\gamma\left(s_{0}\right)-x=\lambda \gamma^{\prime \prime}\left(s_{0}\right)$.

(b) $f_{x}^{\prime}\left(s_{0}\right)=f_{x}^{\prime \prime}\left(s_{0}\right)=0$ if and only if $k_{a}\left(s_{0}\right) \neq 0$ and $x=\gamma\left(s_{0}\right)+\frac{1}{k_{a}\left(s_{0}\right)} \gamma^{\prime \prime}\left(s_{0}\right)$.

(c) $f_{x}^{\prime}\left(s_{0}\right)=f_{x}^{\prime \prime}\left(s_{0}\right)=f_{x}^{\prime \prime \prime}\left(s_{0}\right)=0$ if and only if $k_{a}\left(s_{0}\right) \neq 0, x=\gamma\left(s_{0}\right)+\frac{1}{k_{a}\left(s_{0}\right)} \gamma^{\prime \prime}\left(s_{0}\right)$ and $k_{a}^{\prime}\left(s_{0}\right)=0$.

(d) $f_{x}^{\prime}\left(s_{0}\right)=f_{x}^{\prime \prime}\left(s_{0}\right)=f_{x}^{\prime \prime \prime}\left(s_{0}\right)=0$ and $f^{(4)}\left(s_{0}\right) \neq 0$ if and only if $k_{a}\left(s_{0}\right) \neq 0, x=\gamma\left(s_{0}\right)+$ $\frac{1}{k_{a}\left(s_{0}\right)} \gamma^{\prime \prime}\left(s_{0}\right)$ and $k_{a}^{\prime}\left(s_{0}\right)=0$ and $k_{a}^{\prime \prime}\left(s_{0}\right) \neq 0$.

Proof. (a) By the formula (4.1), $f_{x}^{\prime}\left(s_{0}\right)=0$ if and only if $\gamma^{\prime \prime}\left(s_{0}\right)$ and $\gamma\left(s_{0}\right)-x$ are parallel.

(b) It follows from the formula (4.2) that $f_{x}^{\prime \prime}\left(s_{0}\right)=0$ if and only if $\left|\gamma^{\prime \prime \prime}\left(s_{0}\right), \gamma\left(s_{0}\right)-x\right|=1$, so that $f_{x}^{\prime}\left(s_{0}\right)=f_{x}^{\prime \prime}\left(s_{0}\right)=0$ if and only if $-\lambda k_{a}\left(s_{0}\right)=\mid \gamma^{\prime \prime \prime}\left(s_{0}\right)$, $\lambda \gamma^{\prime \prime}\left(s_{0}\right) \mid=1$ because of (a). The last condition is equivalent to the condition that $k_{a}\left(s_{0}\right) \neq 0$ and $\lambda=-\frac{1}{k_{a}\left(s_{0}\right)}$. 
(c) We also assert that $f_{x}^{\prime}\left(s_{0}\right)=f_{x}^{\prime \prime}\left(s_{0}\right)=f_{x}^{\prime \prime \prime}\left(s_{0}\right)=0$ if and only if $k_{a}\left(s_{0}\right) \neq 0$, $x=\gamma\left(s_{0}\right)+\frac{1}{k_{a}\left(s_{0}\right)} \gamma^{\prime \prime}\left(s_{0}\right)$ and $\left|\gamma^{(4)}\left(s_{0}\right),-\frac{1}{k_{a}\left(s_{0}\right)} \gamma^{\prime \prime}\left(s_{0}\right)\right|=0$. On the other hand, differentiating both sides of the equality $k_{a}\left(s_{0}\right)=\left|\gamma^{\prime \prime}(s), \gamma^{\prime \prime \prime}(s)\right|$, we have $k_{a}^{\prime}(s)=\left|\gamma^{\prime \prime}(s), \gamma^{(4)}(s)\right|$. This implies that $k_{a}^{\prime}\left(s_{0}\right)=0$ if and only if $\left|\gamma^{(4)}\left(s_{0}\right),-\frac{1}{k_{a}\left(s_{0}\right)} \gamma^{\prime \prime}\left(s_{0}\right)\right|=0$.

(d) We have $k_{a}^{\prime \prime}(s)=\left|\gamma^{\prime \prime \prime}(s), \gamma^{(4)}(s)\right|+\left|\gamma^{\prime \prime}(s), \gamma^{(5)}(s)\right|$. Differentiating both sides of the relation $\left|\gamma^{\prime}(s), \gamma^{\prime \prime}(s)\right|=1$, we have $\left|\gamma^{\prime}(s), \gamma^{\prime \prime \prime}(s)\right|=0$. We differentiate again, so that we have an equation $\left|\gamma^{\prime \prime}(s), \gamma^{\prime \prime \prime}(s)\right|+\left|\gamma^{\prime}(s), \gamma^{(4)}(s)\right|=0$. It follows that we have $\mid \gamma^{\prime}(s)$, $\gamma^{(4)}(s) \mid=-k_{a}(s)$. By the formula $(2.1)$ we have $\left|\gamma^{\prime \prime \prime}(s), \gamma^{(4)}(s)\right|=\mid-k_{a}(s) \gamma^{\prime}(s)$, $\gamma^{(4)}(s) \mid=k_{a}(s)^{2}$. Therefore we have $k_{a}^{\prime \prime}(s)=k_{a}(s)^{2}+\left|\gamma^{\prime \prime}(s), \gamma^{(4)}(s)\right|$, so that $k_{a}^{\prime \prime}\left(s_{0}\right)=0$ if and only if $\left|\gamma^{\prime \prime}\left(s_{0}\right), \gamma^{(4)}\left(s_{0}\right)\right|=-k_{a}\left(s_{0}\right)^{2}$. Under the condition (c), $\left|\gamma^{\prime \prime}\left(s_{0}\right), \gamma^{(4)}\left(s_{0}\right)\right|=-k_{a}\left(s_{0}\right)^{2}$ if and only if $\left|\gamma^{(5)}\left(s_{0}\right), \gamma\left(s_{0}\right)-x\right|=-k_{a}\left(s_{0}\right)$. By the formula (4.5), it is equivalent to the condition that $f_{x}^{\prime \prime \prime}\left(s_{0}\right)=0$. This completes the proof.

Remark. If we fix the Euclidean structure on $\mathbb{R}^{2}$ and adopt $t$ as the arclength as in Section 3, we can compare the above results with Fidal's in [7]. He used the function $G(t, x)=\left|x-\gamma(t), \dot{k}(t) T(t)-3 k^{2}(t) N(t)\right|$, so that we have $\frac{\partial F}{\partial s}(s, x)=-\frac{1}{3} k(t)^{-5 / 3} G(t, x)$.

4.2. Affine height functions. Let $S^{\prime}$ be the "unit" circle in $\mathbb{R}^{2}$ given by $S^{1}=\left\{\left(x_{1}, x_{2}\right) \mid x_{1}^{2}+x_{2}^{2}=1\right\}$. We also define a family of smooth functions on $\gamma$ parameterized by $S^{1}$

$$
H: I \times S^{1} \rightarrow \mathbb{R}
$$

by

$$
H(s, u)=\left|\gamma^{\prime}(s), u\right|
$$

We call $H$ the affine height function on $\gamma$.

Differentiating $H(s, u)$ with respect to $s$, we have the following:

$$
\begin{aligned}
& h_{u}^{\prime}(s)=\left|\gamma^{\prime \prime}(s), u\right|, \\
& h_{u}^{\prime \prime}(s)=\left|\gamma^{\prime \prime \prime}(s), u\right|, \\
& h_{u}^{\prime \prime \prime}(s)=\left|\gamma^{\prime \prime \prime \prime}(s), u\right|,
\end{aligned}
$$

where $h_{u}(s)=H(s, u)$ for any $u \in S^{\prime}$.

It follows from these formulae that we have the following proposition.

Proposition 4.2. Let $\gamma: I \rightarrow \mathbb{R}^{2}$ be a plane curve with $\left|\gamma^{\prime}(s), \gamma^{\prime \prime}(s)\right|=1$. Then

(a) $h_{u}^{\prime}\left(s_{0}\right)=0$ if and only if there exists $\lambda \in \mathbb{R}$ such that $u=\lambda \gamma^{\prime \prime}\left(s_{0}\right)$. 
(b) $h_{u}^{\prime}\left(s_{0}\right)=h_{u}^{\prime \prime}\left(s_{0}\right)=0$ if and only if there exists $\lambda \in \mathbb{R}$ such that $u=\lambda \gamma^{\prime \prime}\left(s_{0}\right)$ and $k_{a}\left(s_{0}\right)=0$.

(c) $h_{u}^{\prime}\left(s_{0}\right)=h_{u}^{\prime \prime}\left(s_{0}\right)=h_{u}^{\prime \prime \prime}\left(s_{0}\right)=0$ if and only if there exists $\lambda \in \mathbb{R}$ such that $u=\lambda \gamma^{\prime \prime}\left(s_{0}\right)$ and $k_{a}\left(s_{0}\right)=k_{a}^{\prime}\left(s_{0}\right)=0$.

Proof. (a) By the formula (4.5), $h_{u}^{\prime}\left(s_{0}\right)=0$ if and only if $\gamma^{\prime \prime}\left(s_{0}\right)$ and $u$ are parallel.

(b) It follows from the formula (a) and (4.6) that $h_{u}^{\prime}\left(s_{0}\right)=h_{u}^{\prime \prime}\left(s_{0}\right)=0$ if and only if $-k_{a}\left(s_{0}\right) \lambda=\lambda\left|\gamma^{\prime \prime \prime}\left(s_{0}\right), \gamma^{\prime \prime}\left(s_{0}\right)\right|=\left|\gamma^{\prime \prime \prime}\left(s_{0}\right), u\right|=0$. If $\lambda=0$, then $u=0$. This contradicts the fact that $u \in S^{\prime}$, so that the above condition is equivalent to the condition $k_{a}\left(s_{0}\right)=0$.

(c) Differentiating both sides of the formula $\gamma^{\prime \prime \prime}(s)=-k_{a}(s) \gamma^{\prime}(s)$, we have

$$
\gamma^{(4)}(s)=-k^{\prime}(s) \gamma^{\prime}(s)-k_{a}(s) \gamma^{\prime \prime}(s)
$$

By (b) and (4.7), $h_{u}^{\prime}\left(s_{0}\right)=h_{u}^{\prime \prime}\left(s_{0}\right)=h_{u}^{\prime \prime \prime}\left(s_{0}\right)=0$ if and only if there exists $\lambda \in \mathbb{R}$ such that $u=\lambda \gamma^{\prime \prime}\left(s_{0}\right), k_{a}\left(s_{0}\right)=0$ and $\left|\gamma^{\prime \prime \prime}\left(s_{0}\right), u\right|=-k_{a}^{\prime}\left(s_{0}\right)\left|\gamma^{\prime}\left(s_{0}\right), u\right|=-k_{a}^{\prime}\left(s_{0}\right) \lambda$. Since $\lambda \neq 0$, we have $k_{a}^{\prime}\left(s_{0}\right)=0$. This completes the proof.

We remark that $\lambda(s)= \pm \frac{1}{\sqrt{x_{1}^{\prime \prime}(s)^{2}+x_{2}^{\prime \prime}(s)^{2}}}$, where $\gamma(s)=\left(x_{1}(s), x_{2}(s)\right)$

\section{Unfoldings of functions of one-variable}

In this section we use some general results on the singularity theory for families of function germs. Detailed descriptions are found in the book [5]. Let $F:\left(\mathbb{R} \times \mathbb{R}^{r}\right.$, $\left.\left(t_{0}, x_{0}\right)\right) \rightarrow \mathbb{R}$ be a function germ. We call $f$ an r-parameter unfolding of $f(t)=F\left(t, x_{0}\right)$. The crucial notion is that of a universal unfolding; however, we only use some features of it, so we do not need to give the original definition of universal unfolding (cf., [5]). We say that $f(t)$ has an $A_{k}$ singularity at $t_{0}$ if $f^{(p)}\left(t_{0}\right)=0$ for all $1 \leq p \leq k$, and $f^{(k+1)}\left(t_{0}\right) \neq 0$. In this case we adopt the following definition. Let $j^{k-1}\left(\frac{\partial F}{\partial x_{i}}\left(t, x_{0}\right)\right)\left(t_{0}\right)=\sum_{j=1}^{k-l} \alpha_{j, i} t_{j}$ for $i=1, \ldots, r$, where $j^{k-1}$ denotes the $(k-1)$-jet. We say that $F$ is infinitesimally ( $p$ )versal (respectively, infinitesimally versal) if the $(k-1) \times r$ (respectively, $k \times r)$ matrix of coefficients $\left(\alpha_{j i}\right)$ (respectively, $\left.\left(\alpha_{0 i}, \alpha_{j i}\right)\right)$ has rank $k-1$ (respectively, $k$ ). There are two important sets of unfoldings relative to the above two notions. The bifurcation set of $F$ is the set

$$
\mathcal{B}_{F}=\left\{x \in \mathbb{R}^{r} \mid \text { there exists } t \text { with } \frac{\partial F}{\partial t}=\frac{\partial^{2} F}{\partial t^{2}}=0 \text { at }(t, x)\right\}
$$

and the discriminant set of $F$ is the set

$$
\mathcal{D}_{F}=\left\{x \in \mathbb{R}^{r} \mid \text { there exists } t \text { with } F=\frac{\partial F}{\partial t}=0 \text { at }(t, x)\right\} \text {. }
$$

The proof of Theorem 2.2 is based on the following result. 
Proposition 5.1. (cf., [5]). Let $F:\left(\mathbb{R} \times \mathbb{R}^{r},\left(t_{0}, x_{0}\right)\right) \rightarrow \mathbb{R}$ be an r-parameter unfolding of $f(t)$ which has the $A_{k}$ singularity at $t_{0}$.

(1) Suppose that $F$ is an infinitesimally $(p)$ versal unfolding.

(a) If $k=2$, then $\mathcal{B}_{F}$ is diffeomorphic to $\{0\} \times \mathbb{R}^{r-1}$.

(b) If $k=3$, then $\mathcal{B}_{F}$ is diffeomorphic to $C \times \mathbb{R}^{r-2}$.

(2) Suppose that $F$ is an infinitesimally versal unfolding.

(a) If $k=1$, then $\mathcal{D}_{F}$ is diffeomorphic to $\{0\} \times \mathbb{R}^{r-1}$.

(b) If $k=2$, then $\mathcal{D}_{F}$ is diffeomorphic to $C \times \mathbb{R}^{r-2}$.

Here, $C=\left\{\left(x_{1}, x_{2}\right) \mid x_{1}^{2}=x_{2}^{3}\right\}$ is the ordinary cusp.

Proof of Theorem 2.2 (1). We consider the distance-cubed function $F(s, x)=$, $\left|\gamma^{\prime}(s), \gamma(s)-x\right|$. Using the results of Proposition 4.1 , the bifurcation set of $F$ is precisely the affine evolute of $\gamma$.

Next, the condition for $f=F_{x_{0}}$ to have exactly an $A_{2}$ at $s_{0}$ is for $x_{0}$ to be on the affine evolute of $\gamma$ at $s_{0}$ but $k_{a}^{\prime}\left(s_{0}\right) \neq 0$. Likewise the condition for $f$ to have exactly $A_{3}$ at $s_{0}$ is for $x_{0}$ to be on the affine evolute of $\gamma$ at $s_{0}$ and $k_{a}^{\prime}\left(s_{0}\right)=0, k_{a}^{\prime \prime}\left(s_{0}\right) \neq 0$. By the assumptions (A 1)-(A 3), $f$ has no higher $A_{k}$.

Now $F(s, x)=x_{1}^{\prime}(s)\left(x_{2}(s)-x_{2}\right)-x_{2}^{\prime}(s)\left(x_{1}(s)-x_{1}\right)$, so that we should like to show that $F(s, x)$ is an infinitesimally (p)versal unfolding of $f(s)$ for the above two cases. Here,

$$
\begin{aligned}
& \frac{\partial F}{\partial x_{1}}=x_{2}^{\prime}(s) ; 2 \text {-jet at } s_{0}=x_{2}^{\prime \prime}\left(s_{0}\right) s+\frac{1}{2} x_{2}^{\prime \prime}\left(s_{0}\right) s^{2}, \\
& \frac{\partial F}{\partial x_{2}}=-x_{1}^{\prime}(s) ; 2 \text {-jet at } s_{0}=-x_{1}^{\prime \prime}\left(s_{0}\right) s-\frac{1}{2} x_{1}^{\prime \prime}\left(s_{0}\right) s^{2} .
\end{aligned}
$$

The condition for infinitesimally (p)versal unfolding is given as follows:

(i) When $f$ has $A_{2}$ at $s_{0}$, we require the $1 \times 2$ matrix $\left(x_{2}^{\prime \prime}\left(s_{0}\right),-x_{1}^{\prime \prime}\left(s_{0}\right)\right)$ to have rank 1 , which it always does since $\left|\gamma^{\prime}(s), \gamma^{\prime \prime}(s)\right|=1$.

(ii) When $f$ has $A_{3}$ at $s_{0}$, we require $2 \times 2$ matrix

$$
\left(\begin{array}{cc}
x_{2}^{\prime \prime}\left(s_{0}\right) & -x_{1}^{\prime \prime}\left(s_{0}\right) \\
\frac{1}{2} x_{2}^{\prime \prime}\left(s_{0}\right) & -\frac{1}{2} x_{1}^{\prime \prime}\left(s_{0}\right)
\end{array}\right)
$$

to be nonsingular. But, this just says $k_{a}\left(s_{0}\right) \neq 0$ which is true since $f_{x}^{\prime}\left(s_{0}\right)=f_{x}^{\prime \prime}\left(s_{0}\right)=0$ (cf., Section 4).

Hence infinitesimally (p)versal conditions are automatically satisfied and we have the required result by Proposition 5.1.

Proof of Theorem 2.2 (2). We consider 


$$
\tilde{H}: I \times S^{l} \times \mathbb{R} \rightarrow \mathbb{R}
$$

given by

$$
\tilde{H}(s, u, y)=H(s, u)-y=x_{1}^{\prime}(s) u_{2}-x_{2}^{\prime}(s) u_{1}-y .
$$

The discriminant set of $\tilde{H}$ is

$$
\mathcal{D}_{\bar{H}}^{ \pm}=\left\{\left(\lambda(s) \gamma^{\prime \prime}(s), \lambda(s)\right) \mid \lambda(s)= \pm \frac{1}{\sqrt{x_{1}^{\prime \prime}(s)^{2}+x_{2}^{\prime \prime}(s)^{2}}}, s \in I\right\} .
$$

We only consider $\mathcal{D}_{\tilde{H}}^{+}$. Define a map

$$
\Phi: \mathbb{R}^{2}-\{0\} \rightarrow S^{1} \times \mathbb{R}_{+}
$$

by

$$
\Phi\left(x_{1}, x_{2}\right)=\left(\left(\frac{x_{1}}{\sqrt{x_{1}^{2}+x_{2}^{2}}}, \frac{x_{2}}{\sqrt{x_{1}^{2}+x_{2}^{2}}}\right), \frac{1}{\sqrt{x_{1}^{2}+x_{2}^{2}}}\right)
$$

where $\mathbb{R}_{+}$is the set of all positive real numbers. It is clear that $\Phi$ is a diffeomorphism and $\Phi\left(\right.$ Image $\left.\left(\gamma^{\prime \prime}\right)\right)=\mathcal{D}_{\tilde{H}}^{+}$.

Using the results of Proposition 4.2 and the assumptions (A 1)-(A 3), we only need to consider $A_{1}$ and $A_{2}$ singularities.

We now define a family of functions

$$
F: I \times \mathbb{R}^{2} \rightarrow \mathbb{R}
$$

by

$$
F\left(s, x_{1}, x_{2}\right)=x_{1}^{\prime}(s) \sin x_{1}-x_{2}^{\prime}(s) \cos x_{1}-x_{2}
$$

This is considered as a local representation of $\tilde{H}$. We may use $F$ instead of $\tilde{H}$. Thus

$$
\begin{aligned}
& \frac{\partial F}{\partial x_{1}}=x_{1}^{\prime}(s) \cos x_{1}+x_{2}^{\prime}(s) \sin x_{1}, \\
& \frac{\partial F}{\partial x_{2}}=-1 .
\end{aligned}
$$

The condition for infinitesimally versal unfolding is also as follows:

(i) When $f$ has $A_{1}$ at $s_{0}$, we require the $1 \times 2$ matrix $\left(x_{1}^{\prime}(s) \cos x_{1}+x_{2}^{\prime}(s) \sin x_{1},-1\right)$ to have rank 1 , which it always does. 
(ii) When $f$ has $A_{2}$ at $s_{0}$, we require the $2 \times 2$ matrix

$$
\left(\begin{array}{cc}
x_{1}^{\prime}\left(s_{0}\right) \cos x_{1}+x_{2}^{\prime}\left(s_{0}\right) \sin x_{1} & -1 \\
x_{1}^{\prime \prime}\left(s_{0}\right) \cos x_{1}+x_{2}^{\prime \prime}\left(s_{0}\right) \sin x_{1} & 0
\end{array}\right)
$$

to be nonsingular.

On the other hand,

$$
\left(\cos x_{1}, \sin x_{1}\right)= \pm \frac{1}{\sqrt{x_{1}^{\prime \prime}\left(s_{0}\right)^{2}+x_{2}^{\prime \prime}\left(s_{0}\right)^{2}}}\left(x_{1}^{\prime \prime}\left(s_{0}\right), x_{2}^{\prime \prime}\left(s_{0}\right)\right)
$$

when $s_{0}$ corresponds to $\left(\left(\cos x_{1}, \sin x_{1}\right), x_{2}\right) \in \mathcal{D}_{F}^{ \pm}$, so that $x_{1}^{\prime \prime}\left(s_{0}\right) \cos x_{1}+x_{2}^{\prime \prime}\left(s_{0}\right) \sin x_{1}=$ $\pm \sqrt{x_{1}^{\prime \prime}\left(s_{0}\right)^{2}+x_{2}^{\prime \prime}\left(s_{0}\right)^{2}} \neq 0$. This means that the above matrix is nonsingular. So the infinitesimally versal conditions are automatically satisfied and we have the required result by Proposition 5.1.

\section{REFERENCES}

1. W. BlasChKE, Vorlesungen uber Differentialgeometrie II, Affine Differentialgeometrie (Springer, 1923).

2. J. W. BRUCE, On singularities, envelopes and elementary differential geometry, Math. Proc. Cambridge Philos. Soc. 89 (1981), 43-48.

3. J. W. Bruce and P. J. Giblin, Generic curves and surfaces, J. London Math. Soc. 24 (1981), 555-561.

4. J. W. Bruce and P. J. Giblin, Generic Geometry, Amer. Math. Monthly 90 (1983), 529-545.

5. J. W. Bruce and P. J. Giblin, Curves and singularities (second edition) (Cambridge University Press, 1992).

6. D. L. Fidal and P. J. Giblin, Generic 1-parameter families of caustics by reflexion in the plane, Math. Proc. Cambridge Philos. Soc. 96 (1984), 425-432.

7. D. L. Fidal, The existence of sextactic points, Math. Proc. Cambridge Philos. Soc. 96 (1984), 433-436.

8. P. A. Schirokow and A. P. SChirokow, Affine Differentialgeometrie (Teubner, 1962).

9. B. Su, Affine Differential Geometry (Gordon and Breach, 1983).

DePaRTMENT OF Mathematics

FACULTY OF SCIENCE

HOKKAIDO UNIVERSITY

SAPPORO 060

JAPAN 